\title{
LA JUSTICE ET SON REVERS DANS LES LETTRES PERSANES Thaïs CHAUVEL ${ }^{1}$
}

RÉSUMÉ : Cet article s'interroge sous quelles formes les Lettres persanes de Montesquieu posent la question de la justice et de son revers, l'injustice. On se demande en quoi la richesse de la forme épistolaire, elliptique et diversifiée, permet-elle d'aborder le thème de la justice, de son rapport au pouvoir et à la violence. Pour répondre à cette problématique, on entend analyser l'essai philosophique exposé par le personnage d'Usbek, la fable des Troglodytes employée à des fins didactiques, et enfin, le dispositif fictionnel du "roman du sérail".

MOTS-CLÉS : Lettres persanes ; forme épistolaire ; roman du sérail ; justice ; despotisme; violence;

${ }^{1}$ Master en Lettres Modernes (Université de Sao Paulo) - mail : thais.chauvel@usp.br 


\section{A JUSTIÇA E SEU INVERSO NAS CARTAS PERSAS}

RESUMO: Este artigo investiga sob quais formas as Cartas persas, de Montesquieu, tratam da questão da justiça e de seu revés, a injustiça. Pergunta-se de que modo a riqueza da forma epistolar, elíptica e diversificada, permite abordar o tema da justiça, de sua relação com o poder e a violência. Para responder a essa problemática, propõe-se analisar o ensaio filosófico exposto por Usbek, a fábula dos Trogloditas empregada com fins didáticos e, por fim, o dispositivo ficcional do chamado "romance do serralho"

PALAVRAS-CHAVE: Cartas persas; forma epistolar; romance do serralho; despotismo; justiça; violência

\section{INTRODUCTION}

Charles-Louis de Secondat (1689-1755), baron de la Brède, resté célèbre sous le nom de Montesquieu, est davantage connu pour son ouvrage capital intitulé L'Esprit des lois, paru en 1748. Comme le suggère ce titre, il s'agit d'une œuvre qui s'interroge sur les lois, le droit et la justice. Non sans rapport donc, avec ses études : licencié en droit, Montesquieu est reçu avocat au Parlement de Bordeaux en 1708. Il ne faut cependant pas oublier que ce magistrat, que l'Histoire retient comme l'un des philosophes éminents du XVIIIème siècle, est aussi l'auteur des Lettres persanes, "premier grand texte de la littérature des Lumières" (EHRARD, 1999 : 314). Publiée en mai 1721, cette œuvre de jeunesse de Montesquieu peut être considérée comme "l'ouverture triomphale du temps des philosophes" (OC I, 2004 : 71). L'ouvrage paraît de façon anonyme, car son auteur estime que "si l'on savait qui je suis, on dirait : son livre jure avec son caractère ; il devrait employer son temps à quelque chose de mieux ; cela n'est pas digne d'un homme grave" (p.63), qui occupait déjà une charge de président à mortier au Parlement de Bordeaux.

Dès leur parution, les Lettres persanes remportent "un immense succès à échelle européenne" (OCI, 2004 :16). Ce succès est à la fois foudroyant et durable, puisque l'ouvrage est réédité plus de trente fois jusqu'à la mort de son auteur, en 1755, et nul ne doute de nos jours que "le livre est un chef d'œuvre" (LAUFER, 1961: 188). Toutefois, il semblerait que son importance philosophique soit d'abord passée “inaperçue”, “ses contemporains n'y voyant qu'un badinage romanesque où la fiction orientale était prétexte à une piquante satire de la société française" (ERHARD, 1999: 315). Effectivement, en analysant les rares écrits journalistiques concernant les Lettres persanes, on constate qu'à cette époque "l’intérêt en paraît donc quasi exclusivement satirique" (OC I, 2004 : 63). 
Cela change à la parution de L'Esprit des Lois, publié en 1748 , à Genève. De ce jour, "la solidarité entre les Lettres persanes et L'Esprit des lois est immédiatement affirmée par ceux qui les dénoncent conjointement avec le plus de vigueur, les jansénistes" (OC I, 2004: 64). Raison pour laquelle l'abbé Gaultier publie, en 1751, Les Lettres persanes convaincues d'impiété. Certains critiques du XIXème siècle reprendront le parallélisme entre ces deux œuvres matrices de la pensée de Montesquieu. Ainsi, bien qu'Abel Villemain considère les Lettres persanes "le plus profond des livres frivoles" (VILLEMAIN apud OC I, 2004: 64), il "y reconnaît néanmoins une pensée profonde, dans la mesure où il s'agirait à plusieurs égards d'une première ébauche de L'Esprit des lois" (OCI, 2004 : 64).

Et de fait, en examinant le célèbre mouvement strophique de la préface de Montesquieu à L'Esprit des Lois "si je pouvais faire en sorte que [...], je me croirais le plus heureux des mortels", n'entendons-nous pas l'écho de la lettre (81[83) des Lettres persanes : "si j'étais sûr de suivre [...], je me croirais le premier des hommes” (p.241) ? Pourtant, s'il est vrai que les Lettres persanes abordent certains thèmes, comme celui de la justice, que Montesquieu reprendra par la suite dans L'Esprit des lois, il faut bien convenir que celles-ci sont "plus qu'une préface au grand livre à venir, car un écrivain se révèle dans celui-là” (EHRARD, 1999 : 316, je souligne). De sorte qu'il ne faudrait pas négliger la différence formelle qui distingue ces deux ouvrages, car les Lettres persanes sont bien, d'après Montesquieu lui-même, "une espèce de roman" (p.407).

Le débat concernant l'appartenance des Lettres persanes au genre romanesque se prolonge depuis longtemps et l'avis des critiques diffère encore aujourd'hui. Ce n'est pas une question évidente puisque, comme le remarque Roger Laufer, "au XVIIIème siècle, le genre du roman est d'une infinie souplesse" (Laufer, 1961 : 188). De plus, il s'agit non seulement d'un roman mais, comme son titre l'indique, d'un roman par lettres. Or, l'expression "roman épistolaire" peut être perçue comme anachronique, pour cette raison cet article se réfère plutôt à la forme épistolaire ou encore au dispositif fictionnel et/ou romanesque de l'ouvrage. Et ce, car la forme épistolaire - et donc littéraire, au sens actuel du terme - est au cœur de la problématique de cet article, qui cherche à comprendre comment se présente la réflexion à propos de la justice dans les Lettres persanes. En effet, cette étude se demande en quoi le dispositif épistolaire mis en place par Montesquieu dans son œuvre de jeunesse lui permet-il d'aborder la question de la justice, une constante dans sa vie et dans l'ensemble de son œuvre.

Ainsi, par le biais de la lecture analytique de certaines lettres qui abordent ce thème, cet article s'interrogera, en particulier, sur les ressources spécifiques de ce dispositif épistolaire qui permettent de mûrir une réflexion profonde et novatrice sur la notion de justice. En effet, Montesquieu loue les avantages de la forme épistolaire choisie, en révélant que c'est en partie grâce à cela que " l'auteur s'est donné l'avantage de pouvoir joindre de la philosophie, de la politique et de la morale à un roman » (p.408). En ce sens pluriel et polyphonique, ce « roman par let- 
tres »semble bien appartenir au style rococo français, qui prône la variété. Cet article commencera par présenter l'essai philosophique exposé par le personnage d'Usbek, principal épistolier du roman, pour nourrir ses réflexions métaphysiques à propos de la diversité des pratiques concernant la justice d'un pays à l'autre, et de son rapport à la religion. Par la suite, cette étude entend montrer comment la fable des Troglodytes vient illustrer et confirmer les considérations abstraites d'Usbek à propos de la justice. En effet, si Usbek développe (toujours) ce récit dans un ensemble de quatre lettres, c'est bien à des fins didactiques pour démontrer à son ami Mirza les bienfaits de la vertu, notion qui implique à la fois la sagesse et la tempérance, donc, complémentaire de la justice.

Enfin, cette étude ne saurait ignorer le dispositif romanesque moteur des Lettres persanes, à savoir, le « roman du sérail », qui réunit les missives des femmes, des eunuques et quelques-unes d'Usbek, tous prisonniers d'un lointain harem persan. Tenant compte de cette intrigue orientale, l'article se demande en quoi le dispositif fictionnel développé au cœur du sérail pose la question de la justice à travers son revers, l'injustice. Si bien que l'étude développée dans les pages qui suivent s'interroge sur le rapport entre la justice et la force exposé par le drame du sérail qui «figure en petit ce que le despotisme comme forme de gouvernement contient en grand » (SPECTOR, 2013: 172). L'objectif étant d'identifier ainsi comment la violence et l'abus de pouvoir pratiqués au sein de la fiction du sérail peuvent être liés aux réflexions théoriques et allégoriques exposées dans les Lettres persanes.

Cette première partie cherche à comprendre comment Montesquieu, fidèle au style rococo de son époque, se sert de "la multitude de formes" qu'autorise le dispositif épistolaire (SPECTOR, 2013: 169) mis en place dans cet ouvreur traiter la question de la justice.

Dans un article qui a fait date intitulé "La réussite romanesque et la signification des Lettres persanes de Montesquieu”, Roger Laufer estime que les Lettres persanes sont un "bel exemple de style rococo, auquel appartient la plus grande partie des chefs d'œuvres romanesques du XVIIIème siècle” (LAUFER, 1961 : 193). Cela est vrai dans la mesure où le rococo français, en réaction à l'idéal classique de la forme épurée, préfère la "variété et le mouvement" (LAUFER, 1961: 193). Ce mouvement est effectivement constant dans les Lettres persanes grâce au glissement des point de vues qui s'entrecroisent et des formes narratives diverses qui se succèdent tout au long de l'ouvrage. Jean Starobinski identifie lui aussi le "principe de la variété" des Lettres persanes à la "prédilection de l'art décoratif du rococo pour le changement d'échelle" (STAROBINSKI, 1989 :93). De fait, la discontinuité de ce dispositif épistolaire produit un texte morcelé dans lequel "chaque missive s'impose sa limite" (STAROBINSKI, 1989: 98) aussi bien que sa propre thématique et son style particulier. Ainsi, la souplesse de la forme épistolaire admet à la fois "la lettre insérée dans une autre, fiction rapportée, l'allégorie contée, l'histoire relatée ou l'essai philosophique” (SPECTOR, 2013:169). 
De ce fait, le personnage d'Usbek, principal épistolier du roman, qui participe en parallèle à l'intrigue romanesque qui a lieu dans son sérail abandonné en Perse, élabore par ailleurs des considérations d'ordre métaphysique. Certaines de ses missives ressemblent à des dissertations philosophiques, autant par le sujet qu'elles traitent que par la sérénité de la forme employée. Le curieux Usbek disserte à plusieurs reprises à propos de la justice: il aborde la question des législateurs "des hommes bornés que le hasard a mis à la tête des autres, et qui n'ont presque consulté que leurs préjugés et leurs fantaisie" (76 [129]); du droit public qui, "tel qu'il est aujourd'hui, est une science qui apprend aux princes jusques à quel point ils peuvent viller la justice sans chôquer leur intérêts" (91 [94); et des magistrats qui “doivent rendre la justice de citoyen à citoyen" (92 [95]).

En outre, Usbek examine dans sa lettre 78 [80] les différentes formes de gouvernements. En s'interrogeant sur la proportionnalité de la peine, il remarque que celle-ci s'adapte aussi bien au crime qu'à la coutume du pays où il a été pratiqué : "on punit toujours par degrés, on inflige un châtiment plus ou moins grand à un crime plus ou moins grand" (p. 235). Toutefois, il entend encore démontrer à Rhédi "que dans un État les peines plus ou moins cruelles ne font pas que l'on obéisse plus aux lois". Par conséquent, Usbek estime que "si dans un gouvernement doux le peuple est aussi soumis que dans un gouvernement sévère, le premier est préférable, puisqu'il est plus conforme à la raison" (p. 235). L'article reviendra par la suite sur ce constat, lorsqu'il sera question d'analyser le "roman du sérail”, mais, pour le moment, il importe surtout de ponctuer cet appel d'Usbek à la raison.

Enfin, dans la célèbre lettre 81 (83), Usbek (et peut-être Montesquieu luimême) affirme l'indépendance de la justice humaine par rapport à la croyance en Dieu en déclarant que "libres que nous serions du joug de la religion, nous ne devrions pas l'être de celui de l'équité" (p.240). Cette missive s'ouvre en effet par l'hypothèse suivante: "s'il y a un Dieu, mon cher Rhedi, il faut nécessairement qu'il soit juste”. D'après Ralph Leigh, auteur de l'article "Le thème de la justice dans les Lettres persanes" (1983), cette lettre confirmerait que "Montesquieu est donc déiste [...] son déisme est si hésitant, si indécis qu'il est tout prêt à admettre qu'après tout, à la rigueur, Dieu pourrait bien ne pas exister" (LEIGH, 1983 :208). En situant les Lettres persanes dans leur contexte de production, Ralph Leigh rappelle que le climat "propice à la propagation du relativisme" entraîne le "fléchissement de la foi", celui-ci explique alors, qu'en cette période, "tout se passe comme si on se disait : Dieu n'existe pas, tout est permis” (LEIGH, 1983 :205). Or, la lettre 81(83) marque justement la nécessité de s'élever à l'universel, au-delà même de la diversité des croyances, en affirmant que "quand il n'y aurait pas de Dieu, nous devrions toujours aimer la justice" (p.240).

Qu'est-ce donc que la justice? La lettre 81 (83) en offre la définition suivante : "la justice est un rapport de convenance qui se trouve réellement entre deux choses" (p.239). Toutefois, Usbek reconnaît que "les hommes ne voient pas toujours 
ces rapports ; souvent même lorsqu'ils les voient ils s'en éloignent, et leur intérêt est toujours ce qu'ils voient le mieux" (p.239). Et pourtant, Usbek affirme encore que "nul n'est méchant gratuitement" et que si "les hommes peuvent faire des injustices" c'est uniquement "parce qu'ils ont intérêt de les commettre et qu'ils aiment mieux se satisfaire que les autres" (p.239). La fable des Troglodytes vient illustrer cette hypothèse et complète les conclusions abstraites d'Usbek par un récit qui démontre le risque de privilégier l'intérêt particulier au détriment de l'intérêt commun.

Placée en "situation privilégiée, au seuil du livre" (LEIGH, 1983 : 211), la fable des Troglodytes se déroule sur un ensemble de lettres -assez rares dans les Lettres persanes - plus précisément de la lettre 11 à 14. Ralph Leigh remarque le "ton digne et grave" de ce "prélude thématique" et constate que "cette fable n'est pas un simple élément décoratif, mais un avertissement solennel" (LEIGH, 1983:211) au lecteur. Ce récit, qui répond à la question de Mirza sur l'essence de la vertu humaine (10), est précédé d'une sorte d'épigraphe qu'il convient à présent de citer dans son intégralité :

Pour répondre à ce que tu me prescris, je n'ai pas cru devoir employer des raisonnements fort abstraits ; il y a de certaines vérités qu'il ne suffit pas de persuader, mais qu'il faut encore faire sentir : telles sont les vérités de morale ; peutêtre que ce morceau d'histoire te touchera plus qu'une philosophie subtile. (11, p. 82)

Contrairement aux "raisonnements fort abstraits" présentés dans les lettres mentionnées ci-dessus, Usbek préfère reconstituer ici "ce morceau d'histoire" car, explique-t-il, "il y a certaines vérités qu'il ne suffit pas de persuader, mais qu'il faut encore faire sentir". Sa formule oppose le verbe "persuader" associé à "une philosophie subtile", au verbe "toucher" et à l'expression "faire sentir". Alors que le verbe persuader, synonyme de convaincre, se sert d'arguments logiques pour "amener quelqu'un à croire quelque chose" (Trésor de la Langue française), le verbe "toucher", au sens figuré, signifie "émouvoir en suscitant des sentiments d'affection" (Trésor de la Langue française), ce qui correspond bien à l'expression "faire sentir". Ainsi, s'il est vrai que, par la suite, Usbek n'hésitera pas à développer sa "philosophie subtile" à partir de "raisonnements forts abstraits", il préfère reconstituer ici une parabole, afin de provoquer une impression affective (c'est-àdire, qui n'est pas uniquement rationnelle) chez le lecteur pour mieux lui "faire sentir" une vérité morale abstraite. Si l'on considère le processus de doubleénonciation constitutif du roman par lettres, cette explication à finalité didactique d'Usbek peut aussi être perçue comme une affirmation de Montesquieu l'égard de ses propres lecteurs. Dès lors, cette espèce d'épigraphe ne vaudrait pas exclusivement pour la fable des Troglodyte mais aussi pour l'ensemble de l'œuvre. 
Ce récit, encadré par les lettres qui composent le "roman du sérail”, ressemble au conte philosophique, par sa forme narrative, sa brièveté (il compte dix pages dans l'édition de Philip Stewart, 2013), et, enfin, par la leçon qui s'en dégage. De ce fait, l'histoire commence par reprendre la formule traditionnelle du conte populaire (dont le conte de fée est une variante) : "Il y avait en Arabie un petit peuple appelé Troglodyte, [...] qui, si nous en croyons les historiens, ressemblaient plus à des bêtes qu'à des hommes [...] ils étaient si méchants et si féroces qu'il n'y avait parmi eux aucun principe d'équité ni de justice" (11, p. 82).

La lettre 11 démontre par une série d'exemples "comment les Troglodytes périrent par leur méchanceté même, et furent les victimes de leurs propres injustices" (12, p. 86). En effet, des désastres naturels marqués par l'image de l'excès - la sécheresse et l'inondation -accablent les Troglodytes en engendrant la famine. Puis, ceux qui y survivent, doivent alors souffrir d'une "cruelle maladie". De façon indirecte, ce peuple se donne lui-même la mort, puisqu'il refuse de payer le salaire d'un "habile médecin" (p. 85) qui aurait pu soigner le mal. Le sermon de celui-ci est on ne peut plus clair: "hommes injustes, vous avez dans l'âme un poison plus mortel que celui dont vous voulez guérir; vous ne méritez pas d'occuper une place sur la terre, parce que vous n'avez point d'humanité, et que les lois de l'équité vous sont inconnues" (p.85).Ainsi, c'est bien au prix de leur vie, "que s'achète, chez les Troglodytes, le souci exclusif de l'intérêt personnel” car, comme l'explique Jean Starobinski, "le peuple ne peut survivre en tant que communauté, le revers de l'anarchisme égoïste, c'est l'anéantissement collectif' (STAROBINSKI, 1989:119).

La fable des Troglodytes pourrait s'achever sur cette morale. Or, la lettre 12 poursuit le récit en racontant qu'“il y avait dans ce pays deux hommes bien singuliers : ils avaient de l'humanité, ils connaissaient la justice, ils aimaient la vertu [...] ils travaillaient avec une sollicitude commune pour l'intérêt commun ;" (p. 86). Non seulement ces deux hommes étaient naturellement vertueux, mais ils s'efforçaient "d'élever leur enfants à la vertu". Pour cela, ils montrent aux enfants "l'exemple si touchant", au sens d'émouvant, du malheur de "leurs compatriotes indignes" à des fins didactiques. Tout comme Usbek (et Montesquieu aussi), "ils leur faisaient surtout sentir que l'intérêt de particuliers se trouve toujours dans l'intérêt commun" (p. 87).

"Je ne saurais assez te parler de la vertu des Troglodytes", déclare Usbek à Mirza dans la lettre 13. Celle-ci se présente en quelque sorte comme le revers de la lettre 11, puisque l'auteur y développe une quantité d'exemples positifs qui montrent en quoi la nouvelle génération des Troglodytes respecte cette vérité fondamentale selon laquelle "la justice pour autrui est une charité pour nous" (p.87). Cependant, la prospérité du nouveau peuple Troglodyte attire bien sûr l'envie des peuples voisins qui "entrèrent armés dans la terre des Troglodytes". (p. 90). L'auteur raconte alors "le combat de l'injustice et de la vertu ; ces peuples lâches qui ne cherchaient que le butin, n'eurent pas même honte de fuir, et ils cédèrent à la vertu des Troglodytes sans en être touchés" (p. 91). 
Ne serait-il pas possible d'entrevoir, dans la fable des Troglodytes et la défaite de tous ceux qui n'ont pour but que leur intérêt personnel exclusif, les principes qui seront immortalisés par la morale Kantienne ? La vérité essentielle démontrée par cette parabole, à savoir, "que la justice pour autrui est une charité pour nous", ne résonne-t-elle pas dans le fondement 105 de Kant: "Agis de telle sorte que tu traites l'humanité aussi bien dans ta personne que dans la personne de tout autre toujours en même temps comme une fin, et jamais simplement comme un moyen"?

Pourtant, la fable des Troglodytes ne s'achève pas vraiment sur la victoire de la vertu sur l'injustice, car la lettre 14 introduit la thématique du pouvoir. À mesure que les Troglodytes croient et prospèrent, ils décident de "choisir un roi", parmi eux celui qui est "le plus juste" (14, p.91). Or, ce "vieillard vénérable" à qui l'on veut "déférez la couronne" leur dit dans un registre pathétique "comptez que je mourrai de douleur d'avoir vu en naissant les Troglodytes libres, et de les voir aujourd'hui assujettis" (p.91). Cette fin laisse bien entendre toute la difficulté d'être vertueux malgré soi, qu'il est parfois plus aisé de se soumettre aux lois d'un prince, que d'obéir à la vertu - cette disposition ferme, qui porte à faire le bien et à fuir le mal. Le sage formule alors la question suivante : "comment se peut-il que je commande quelque chose à un Troglodyte? Voulez-vous qu'il fasse une action vertueuse parce que je la lui commande, lui qui la ferait tout de même sans moi, et par le seul penchant de la nature ?" (p.92) et c'est avec peine qu'il conclut qu'accepter la couronne, symbole du pouvoir, serait laissé le peuple "sous un autre joug que celui de la vertu" (p. 92). Sur ces mots graves et solennels s'achève alors la fable des Troglodytes.

Il convient maintenant de comparer la vertu libre vantée par l'allégorie contée des Troglodytes à la vertu qu'Usbek veut imposer à son sérail en tant que maitre et époux. Dans la lettre 19 (20), qui n'est pas si éloignée de la fable des Troglodytes, Usbek reprend à trois reprises le mot vertu. Toutefois, au ton impérieux de cette lettre, on peut se demander si le terme évoqué renferme bien le même sens que chez les Troglodytes qu'il vient d'évoquer. En effet, dans la sévère lettre qu'il adresse à son épousez chi, Usbek lui fait le reproche suivant : "Vous vous vantez d'une vertu qui n'est pas libre » (19 [20], p.102). Ainsi, comme le remarque Philip Stewart, "sa propre rhétorique le trahit », puisqu'Usbek semble bien « se rendre compte que la situation incarne une contradiction : ses femmes sont vertueuses dans la mesure où elles sont forcées de l'être, ce qui prouve peu » (STEWART, 1999: 143). Dans son sérail, Usbek n'occupe-t-il pas la place que redoutait tant le vénérable vieillard de la lettre 14, lequel constatait sagement que, si les Troglodytes étaient " assujettis » aux lois d'un prince, ils vivraient alors « sous un autre joug que celui de la vertu » (14, p. 92)?

Le mot « vertu » apparaît 35 fois sous la plume d'Usbek dans les Lettres persanes (EHRARD, 1999 : 322), mais il semble se dénaturer lorsqu'il est employé dans les missives destinées au sérail, où il permet de justifier la « servitude domesti- 
que ». C'est d'ailleurs ce que lui reproche son épouse Roxane dans la lettre finale : «j'ai profané la vertu en souffrant que l'on appelât de ce nom ma soumission à tes fantaisies » (150 [161], p. 406) D'après l'analyse de Jean Starobinski, « c'est là une nouvelle mise en garde que Montesquieu nous adresse: il est des mots trop grands, trop abstraits ou trop vagues, pour que l'on ne désigne pas, par leur truchement, les choses les plus dissemblables " (STAROBINSKI, 1989: 111). À cet égard, Christophe Martin interprète cette " répétition obsédante » (MARTIN, 2013 : 19) de vocables abstraits tels que «pudeur », « innocence » et «nature », ainsi que la "scansion permanente du pronom d'adresse "vous » " (MARTIN, 2013 : 19) (alors que ses épouses le tutoient), comme « autant de signes d'une impuissance radicale du discours » (MARTIN, 2013:19).

En déjouant d'avance la défense de Zachi «Vous me direz peut-être que vous m'avez toujours été fidèle. Eh pouviez-vous ne l'être pas?», Usbek rend compte de la situation d'enfermement de ses femmes par le biais d'interrogations rhétoriques telles que celles-ci : «Comment auriez-vous trompé la vigilance de ces eunuques noirs [...]? Comment auriez-vous pu briser ces verrous et ces portes qui vous tiennent enfermée?» (19 [20], p.102).À ce propos, Céline Spector constate que « loin de permettre aux épouses d'échapper aux tentations du vice, le harem et son modus operandi (emprisonnement des femmes, surveillance des eunuques, discipline rigoureuse), leur interdit toute vertu, leur clôture étant la matrice de toutes les perversions » (SPECTOR, 2013: 173). Usbek le reconnaît lui-même en partie, dans la mesure où il perçoit, dès la lettre 19 (20), que la sincérité de ses femmes, « comme le désir d'être fidèle, dépendait en fait d'une liberté d'agir qui leur est refusée » (VOLPILHAC-AUGER, STEWART, $2004: 57$ ).

"L’on s'accorde en général à considérer le sérail dans les Lettres persanes comme une figure en réduction du despotisme : la servitude domestique valant comme image érotisée de la servitude politique » (MARTIN, $2013: 11)$. Dans un article du Dictionnaire Montesquieu, Bertrand Binoche explique que «dépourvu de toute règle juridique en général, le despotisme est dépourvu de lois fondamentales [...] de là résulte que les seules contrainte stables qui subsistent sont nécessairement infra-juridiques » (BINOCHE, 2013). Effectivement, lorsqu'Usbek réprimande Zachi «parce que vous faites quelque chose que les lois du sérail vous défendent », il entend par là que la loi du sérail équivaut à des " préceptes théologiques» qui appartiennent à la loi coranique (SPECTOR, 2013:174) - donc spécifique à une croyance, et non pas universelle. De fait, Usbek emprunte certaines expressions au vocabulaire religieux pour désigner le sérail, telles que : «lieu sacré», « temple sacré», « mœurs saintes».

À la chute du roman (150 [161]), Roxane, l'épouse préférée d'Usbek, lui annonce : «j'ai pu vivre dans la servitude, mais j'ai toujours été libre ; j’ai réformé tes lois sur celles de la nature, et mon esprit c'est toujours tenu dans l'indépendance» (p. 406). Les lois de pudeur et de modestie, calquées sur la loi coranique qu'Usbek impose au sérail sont aussi particulières que subjectives ( tes 
lois »), elles n'appartiennent pas à « la justice éternelle » qui « ne dépend point des conventions humaines » (p. 240) et qu'Usbek défendait avec ardeur dans la lettre 81 (83). Usbek, auteur de la fable des Troglodytes, qui sait donc « que les hommes deviennent injustes dès qu'ils " préfèrent leur propre satisfaction à celle des autres ", est lui-même incapable d'apercevoir sa propre injustice » (STAROBINSKI, 1989: 120).Il témoigne néanmoins d'une certaine lucidité en doutant de sa capacité à être juste: " Oui, Rhédi, si j'étais sûr de suivre toujours inviolablement cette équité que j'ai devant les yeux, je me croirais le premier des hommes » (81 [83], p. 241). Pour Jean Starobinski, ce personnage « clivé » (STAROBINSKI, 1989: 108) est l'exemple poignant « d'une séparation persistante entre le domaine de la réflexion et celui des actes » (STAROBINSKI, 1989: 120).

De cette façon, Usbek, tout en étant l'auteur d'une admirable théorie à propos de la justice, ne peut pas pour autant s'empêcher d'avoir une perspective partielle lorsqu'il s'agit du sérail dont il est le possesseur. De surcroît, Usbek est dévoré, ditil dès la lettre 6, par « une jalousie secrète » (p.72). Non seulement sa perspective est partielle, mais, en plus, il est absent de son harem pendant l'intégralité de l'œuvre. Il faut rappeler, en effet que la « donnée fondatrice du dispositif épistolaire » (MARTIN, 2013 : 11) est bien le départ d'Usbek sur lequel s'ouvre le roman. L'éloignement de ce personnage est l'élément déclencheur fictionnel qui démarre la correspondance du « roman du sérail » : c'est précisément parce qu'il n'est pas dans son sérail qu'Usbek envoie des lettres à ses femmes et surtout à ses eunuques.

Il dépend d'ailleurs de ces-derniers pour saisir ce qui se passe à l'intérieur de « ces portes fatales» (2, p. 66). Car « c'est à travers le filtre de leur jugement, de leurs craintes ou de leurs désirs de vengeance contre des épouses nécessairement haïes qu'il accède aux évènements formant la trame lacunaire et incertaine de la chronique du sérail »(MARTIN, 2013: 17). Or, étant donné que « la loi du sérail se réduit au pouvoir absolu du maitre, pouvoir de vie et de mort que reconnaissent nécessairement toutes les femmes et tous les eunuques dont on entend la voix dans le roman » (VOLPILHAC-AUGER, STEWART, 2004 : 57), il est évident qu' " Usbek (ni le lecteur) ne saurait évidement accorder une confiance aveugle» (MARTIN, 2013: 17) aux lettres provenant du sérail.

En effet, les épouses sont parfaitement conscientes que « la loi du sérail se réduit au pouvoir absolu du maître » (VOLPILHAC-AUGER, STEWART, $2004: 57$ ). En tant que maître du sérail - rôle dont il semble être las dès la lettre 6 - Usbek concentre à lui seul tous les pouvoirs, car il fait la loi, juge (à distance et d'après le récit subjectif des eunuques) des infractions commises au sein de cette " affreuse prison » (9), et exerce, enfin, un droit de vie et de mort sur les membres de son sérail. Selon l'expression de Pierre Testud, "chaque lettre nous enferme dans la subjectivité de son auteur », ainsi, « nous n'avons, sur les événements qu'une vision fragmentaire » (TESTUD, 1966 : 647). À cela, il faudrait encore ajouter que l'identité du destinateur, comme celle du destinataire, ainsi que leurs statuts res- 
pectifs influencent bien entendu le contenu de chaque lettre, qui correspond davantage à l'intérêt personnel de chacun, ne rendant pas forcément compte de la « vérité référentielle» (STEWART, 1999 :143). Raison pour laquelle Philip Stewart met en garde contre une «tendance très rependue [...] à lire les missives comme de fiables déclarations d'états de faits» (STEWART, 1999: 142), puisque « la forme épistolaire qui insère tous les personnages dans ce système de pouvoir, empêche toute énonciation libre et sincère» (VOLPILHAC-AUGER, STEWART, 2004: 57).

La lettre 4, de Zephis, dont Philip Stewart constate « l'ambiguïté totale » (1999: 144), le démontre bien. La deuxième femme d'Usbek raconte dans cette missive comment «ce monstre noir » (p.70), c'est-à-dire l'eunuque, suspicieux des rapports qu'entretient Zephis avec son esclave Zelide, veut «à toute force » lui ôter son esclave. Zephis répond en accusant son accusateur d'avoir des "soupçons extravagants », mais refuse de se défendre de cette accusation « déshonorante » déclarant tout simplement que : «j'ai trop de respect pour moi-même pour descendre à des justifications » (p.71). Elle affirme n'admettre «d'autre garant » de sa conduite qu'Usbek, qui est déjà loin. Cette lettre est d'autant plus intéressante car Zephis y présente elle-même le point de vue de l'eunuque qui n'est pas représenté dans une lettre à part. Cette missive renferme ainsi deux perspectives radicalement contradictoires et le lecteur avertit peut donc se demander lequel des deux ment. Il convient de se rappeler que le droit romain exige, pour déterminer une condamnation, qu'il y ait deux témoins ou alors la confession du coupable (JANINE RIBEIRO, 1994: 71). Pour juger cette affaire en accord avec le droit romain, il faudrait donc qu'Usbek ait un deuxième témoignage pour confirmer celui de l'eunuque, ou bien que Zephis s'avoue coupable. Cette lettre restera sans réponse mais le lecteur apprend à la lettre 19 (20) que Zelide est passée au service de Zachi, puis est devenue l'esclave de Zelis (51 [53]), il peut en conclure ainsi l'eunuque est bien parvenu à séparer Zephis de son esclave.

La célèbre polyphonie des Lettres persanes va de pair avec la polygamie du sérail. En réunissant les lettres écrites par les femmes, les plaçant les unes à la suite des autres au début (3, 4, et 7), puis au dénouement (148, L.S. 9 [157], L.S. 10 [158]), un effet qui renforce leur nombre se produit. En reprenant la remarquable analyse d'Alain Grosrichard, on peut comprendre que cette féminité figurée dans les lettres « se donne comme une multiplicité indénombrable d'exemplaires quasi interchangeables » (GROSRICHARD, 1979 : 177). La répétition de l'initiale $Z$ dans les prénoms de Zachi, Zelis et Zephis, renforce également la multiplicité féminine face à Usbek, qui se caractérise par son unicité. Or, comme le démontre Alain Grosrichard, " le rapport du supérieur à l'inférieur tend à se donner toujours comme rapport de l'Un au multiple » (GROSRICHARD, 1979 : 182). Et, de fait, on remarquera que des cinq lettres qu'Usbek écrit à ses femmes, deux d'entre elles s'adressent collectivement aux épouses (63 [65] et 146 [154), ce qui contribue à dévaluer le féminin, dilué dans le multiple. 
Cet entrecroisement de voix féminines dans le roman du sérail permet de juxtaposer les regards sur un même épisode, comme celui de la punition doublement appliquée à Zachi et Zelis. À ce propos, il faut rappeler que seule la lettre de Roxane ce référait à cet événement dans l'édition de 1721, qui y était rapporté de la façon suivante : "Zachi et Zelis ont reçu dans leur chambre, dans l'obscurité de la nuit, un traitement indigne : le sacrilège n'a pas craint de porter sur elles ses viles mains » (p. 401). Par la suite, Montesquieu écrit les lettres supplémentaires L.S. 9 et L.S. 10, qui viennent enrichir le dénouement en exposant les réactions respectives des victimes. La plainte de Zachi permet de découvrir quel est le « traitement indigne» mentionné auparavant par Roxane : «Un barbare m'a outragée jusque dans la manière de me punir ! Il m'a infligé ce châtiment qui commence par alarmer la pudeur, ce châtiment qui ramène, pour ainsi dire, à l'enfance » (L.S. 9 p. 402). D’après Catherine Volpilhac-Auger, "Zachi évoque, sans le nommer pourtant, le fouet ou fessée, " punition des enfants» selon Rousseau, mais ici punition orientale qui ne s'auréole chez Montesquieu d'aucune connotation érotique» (VOLPILHAC-AUGER, $2013:$ 122). Zelis, elle, s'indigne qu' « à mille lieues de moi vous me jugez coupable ; à mille lieues de moi vous me punissez» (L.S.10, p. 403), elle lui reproche donc exercer à l'aveugle son pouvoir absolu. Zelis traite alors ouvertement Usbek de tyran, comprenant qu' « un eunuque barbare porte sur moi ses viles mains, il agit par votre ordre : c'est le tyran qui m'outrage, et non pas celui qui exerce la tyrannie » (p.403).

En effet, « comme le despote, le maitre du sérail ne manifeste jamais son pouvoir en personne mais toujours par son représentant ou son supplément: l'eunuque » (MARTIN, 2013: 23). Christophe Martin rappelle «qu'il est dans la nature du despotisme de déléguer tous les pouvoirs à un vizir ", de ce fait, " en quittant son sérail et en déléguant entièrement son pouvoir aux eunuques, Usbek offre l'image achevée du parfait despote, maître absent par définition, dont le fantôme menaçant entretient la terreur parmi les esclaves et les épouses » (MARTIN, 2013 : 23).Cette terreur, Usbek l'exerce par le recours à la violence effective ou métaphorique. Même s'il paraît peu enclin à sévir, il exécute l'eunuque Nadir, «qui paiera de sa tête son infidélité et sa perfidie» dès 1712. De même, il semble accorder peu d'importance à la vie de ses esclaves : " qui êtes-vous, que de vils instruments que je puis briser à ma fantaisie, qui n'existez qu'autant que vous savez obéir, qui n'êtes dans ce monde que pour vivre sous mes lois ou pour mourir dès que je l'ordonne» (20 [21], p.105).

Toutefois, Usbek semble d'abord enclin à la modération, lorsque le premier eunuque lui écrit, en 1714, pour peindre les désordres qui se produiraient dans le sérail, Usbek se borne à écrire une lettre collective à ses femmes. Il déclare dans sa lettre (63 [65]) « Je ne sais me servir de ces moyens violents que lorsque j'ai tenté tous les autres : faites donc en votre considération ce que vous n'avez pas fait pour la mienne». À la suite de cette menace voilée, il leur implore presque : « changez donc de conduite, je vous prie». Le ton de cette lettre est cohérent avec la con- 
fession faite à Nessir dans la lettre 6 : «n'aimerais-je pas mille fois mieux une obscure impunité qu'une correction éclatante? »(p.73).On devine dans la formule « je vous prie » qui suit de près l'utilisation de l'impératif, un discours « sans prise sur le réel » (MARTIN, $2013:$ 19).

Cela s'aggrave par la suite lorsque les lettres 140 (148) et 142 (150), contenant des « ordres prompts et violents » (p. 394) destinés au Premier eunuque, se perdent. Ainsi, la parole d'Usbek «qui rêve d'accomplir ce qu'elle profère » (MARTIN, 2013 : 19) à l'instar d'une lettre de cachet, n'est bel et bien qu'une lettre morte. Christophe Martin remarque le «contraste cruel entre la lenteur de l'échange épistolaire et la logique despotique de l'instantanéité» (MARTIN, 2013: 18). Cet ainsi que « l'échange épistolaire se révèle parfaitement contradictoire avec l'économie despotique de l'instantanéité ; » (MARTIN, 2013: 19), figurée par le « clin d'œil » qui est censé envoyé la mort (GROSRICHARD, 1979:80).

Les lettres dans lesquelles Usbek ordonne de sévir mêlent le vocabulaire de la justice au registre de la violence, il parle de «tribunal redoutable », de " punitions et châtiments » (p.395), de « crimes » (p. 397). Il donne carte blanche à Solim pour qu'il organise une violente répression : "Je te mets le fer à la main. Je te confie ce que j'ai à présent dans le monde de plus cher, qui est ma vengeance" (p.397), lui disant bien : "extermine les coupables, et fais trembler ceux qui se proposeraient de le devenir” (p.398). Dans la lettre 149 (159), Solim, qui semble avoir compris que la lenteur de l'échange épistolaire est incompatible avec la structure despotique du sérail, exprime ainsi son hésitation: "Je ne sais si j'attendrai, sublime seigneur, tes ordres sévères ; tu as mis ta vengeance en mes mains, je ne dois pas la faire languir" (149 [159, p. 404). Finalement, Solim se décide le jour même "Je vais punir" (L.S. 11 [160], p.405), annonce-t-il. Il imagine une punition sanguinaire dans l'enceinte du sérail, se figurant "tout le sang que je vais y rependre", et emploie la première personne du pluriel pour s'allier à Usbek : "mon âme et la tienne vont s'apaiser, nous allons exterminer le crime" (L.S.11 [160], p. 405). On constate que Solim reprend les formules utilisées par son maître, telles que "vengeance", "crime" et le verbe "exterminer".

Par son dénouement tragique, le dispositif épistolaire et fictionnel du "roman du sérail” pose, autant par la forme que par le développement de la diégèse, la question du rapport de la justice au pouvoir et du recours à la violence. En permettant de concilier "diversité et subjectivité », la forme épistolaire " rend compatibles la pluralité des voix et l'emploi de la première personne » (TESTUD, 1966 : 646). La ressource du «je » est essentielle pour que le lecteur s'identifie aux personnages de l'intrigue. D'autre part, la polyphonie de ce roman par lettres va de pair avec le relativisme. Enfin, par son écriture elliptique, tout n'est pas dit dans la trame du sérail, et cette forme fragmentaire, invite le lecteur à compléter les lacunes et à comprendre ainsi tout le sens de l'intrigue « roman du sérail ». 
Cet article a tenté de montrer sous quels aspects se construit la réflexion sur la justice dans les Lettres persanes, de Montesquieu. En explorant au maximum les ressources de la forme épistolaire, l'auteur crée une ouvre plurielle qui lui permet d'aborder la question de la justice, de son rapport à la religion et au pouvoir, de différentes façons. De l'essai philosophique, évoquant des raisonnements abstraits, aux événements tragiques du "roman du sérail" qui viennent renverser tous les propos raisonnables élaborés par Usbek, en passant par la fable des Troglodytes, qui expose allégoriquement la priorité de l'intérêt commun sur l'intérêt particulier, Montesquieu développe le thème de la justice sous toutes ses formes. De façon à la fois romanesque et philosophique, Montesquieu démontre la nécessité de s'élever à l'universel aussi bien que l'importance de la Raison. La chute du "roman du sérail", qui "permet de déborder sur la conjoncture historique présente", s'achève, par la révolte de Roxane sur son oppresseur, sur la nécessité de la transgression. Si l'allégorie contée des Troglodytes montre, dans un premier temps, que ce sont les interdictions qui rendent possibles la vie en société, la diégèse et surtout la fin tragique du "roman du sérail" démontrent comment la transgression de certaines règles est également indispensable à l'invention de la liberté.

RÉFÉRENCES BIBLIOGRAPHIQUES

BINOCHE, B. «Despotisme ». In : Dictionnaire Montesquieu, dira. Catherine Volpilhac-Auger, ENS de Lyon, 2013 (setembro). Disponívelem :<http://dictionnaire-montesquieu.ens-lyon.fr/fr/article/1367168359/fr >.

EHRARD, J. ; POMEAU, R. Histoire de la littérature française : de Fénelon à Voltaire. Paris : Flammarion, 1998.

GROSRICHARD, A. Structure du sérail : la fiction du despotisme asiatique dans l'Occident classique. Paris : Seuil, 1979.

LAUFER, R. " La réussite romanesque et la signification des Lettres persanes de Montesquieu ». Revue d'histoire littéraire de la France, vol. 61, $\mathrm{n}^{0}$ 2, 1961. pp. 188-203.

Disponívelem :<http://gallica.bnf.fr/ark:/12148/bpt6k5727838m/f38.image>.

LEIGH, M. R. A. «Le Thème de la justice dans les Lettres persanes ». Cahiers de l'Association internationale des études françaises, 1983, vol. 35, pp. 201-219. Disponíve- 
lem :<http://www.persee.fr/web/revues/home/prescript/article/caief_05715865_1983_num_35_1_2413>.

MARTIN, C. Usbek in absentia ou le sérail sans maître. In : STEWART, P. Les Lettres persanes en leur temps. Paris : Classiques Garnier, 2013.

MONTESQUIEU. Lettres persanes. Paris : Classiques Garnier poche, 2013.

RIBEIRO, R. J. «A máscara, o véu, o coração ou Um erro de Montesquieu, sobre o harém, nas Cartas persas». In :Arte pensamento. São Paulo : Cia. Das Letras, 1994. pp. 69-90.

STAROBINSKI, J. Exil, satire tyrannie : les Lettres persanes. In : Le Remède dans le mal. Critique et légitimation de l'artifice à l'âge des Lumières. Paris : Gallimard, 1989.

STEWART, P. Toujours Usbek. Eighteenth-Century Fiction, vol.11, n ${ }^{0}$ 2, 1999.pp. 141-150.

SPECTOR, C. Le despotisme des passions dans les Lettres persanes. In : MARTIN, C. Les Lettres persanes de Montesquieu. Paris : PUPS, 2013.

TESTUD, P. Les lettres persanes, roman épistolaire. $R H L F$, vol. $66, \mathrm{n}^{\circ} 4,1966$. pp. 642-656.

Disponívelem :<http://gallica.bnf.fr/ark:/12148/bpt6k5726275h/f52.image>.

VOLPILHAC-AUGER; STEWART. Introduction : pour une "histoire véritable" des Lettres persanes. In : MONTESQUIEU, Euvres complètes I, Lettres persanes. Oxford : Voltaire Foundation \& Napoli : Istituto italiano per glistudifilosofici, 2004. 